Comptes Rendus Physique 8 (2007) 598-608

\title{
Temporal ordering of nonequilibrium fluctuations as a corollary of the second law of thermodynamics
}

\author{
Pierre Gaspard \\ Center for Nonlinear Phenomena and Complex Systems, \\ Université Libre de Bruxelles, Code Postal 231, Campus Plaine, B-1050 Brussels, Belgium
}

\begin{abstract}
In nonequilibrium steady states, the probabilities of the time-reversed paths differ in general from the path probabilities and they decrease at different rates respectively given by the time-reversed and standard entropies per unit time. These quantities characterize temporal disorder in the time-reversed paths and the paths themselves. The difference between these quantities gives the thermodynamic entropy production in units of Boltzmann's constant. Out of equilibrium, the temporal disorder of the time-reversed paths turns out to be larger than the temporal disorder of the paths themselves. In this way, a theorem is obtained according to which the temporal order of the paths is greater than for the time-reversed paths. The temporal disorder or dynamical randomness of the paths and their time-reversal have large-deviation properties directly connected to the thermodynamic entropy production.
\end{abstract}

\section{Résumé}

Ordonnancement temporel des fluctuations de non-équilibre comme corollaire de la seconde loi de la thermodynamique. Dans les états stationnaires de non-équilibre, les probabilités des chemins renversés dans le temps diffèrent en général des probabilités des chemins et elles décroissent à des taux donnés par les entropies par unité de temps, respectivement réverse et standard. Ces quantités caractérisent le désordre temporel des chemins renversés dans le temps et des chemins eux-mêmes. La différence entre ces quantités donne la production d'entropie thermodynamique en unités de la constante de Boltzmann. Hors d'équilibre, il ressort que le désordre temporel des chemins renversés dans le temps est plus grand que le désordre temporel des chemins eux-mêmes. Ainsi, un théorème est obtenu selon lequel l'ordre temporel des chemins est plus grand que pour les chemins renversés dans le temps. Le désordre temporel ou hasard dynamique des chemins et des chemins réverses ont des propriétés de grandes déviations directement reliées à la production d'entropie thermodynamique.

Key words: Thermodynamic entropy production; dynamical randomness; entropy per unit time; time reversal.

Mots-clés : Production d'entropie thermodynamique; hasard dynamique; entropie par unité de temps ; renversement du temps.

Email address: gaspard@ulb.ac.be (Pierre Gaspard). 


\section{Introduction}

The second law of thermodynamics states that the entropy of an isolated system increases with time as the thermodynamic equilibrium is approached. Since Boltzmann, entropy is known to characterize disorder in the instantaneous statistical distribution of the microscopic degrees of freedom. If the system is spatially extended, the thermodynamic entropy per unit volume is a measure of spatial disorder, which thus tends to increase with time in an isolated system. If the system is open, entropy can be carried from the system to the environment, allowing the spatial ordering into equilibrium structures such as crystals which are formed by the process of cooling [1]. In far-from-equilibrium open systems, spatially ordered dissipative structures such as chemical Turing patterns can be maintained by energy supply [2]. In such patterns, some chemical species are spatially separated which reduces Gibbs' mixing entropy of these species.

Beside spatial disorder, we may be interested in the property of temporal disorder, also called dynamical randomness. During the last two decades, advances in dynamical systems theory have shown how this property can be characterized in terms of the Kolmogorov-Sinai (KS) entropy per unit time $[3,4,5]$. It is the analogue of the thermodynamic entropy per unit volume, but for disorder in time instead of space. The KS entropy per unit time is positive in the chaotic regimes, which is the case in turbulence for macroscopic dissipative systems. The concepts of dynamical systems theory have also been applied to microscopic systems and, in particular, the ones of statistical mechanics [6]. The KS entropy per unit time has been calculated in systems of interacting particles, showing their chaoticity for typical interaction potentials at high enough temperature $[7,8]$. This microscopic chaos is the irregular motion of the particles due to their incessant collisions with each others and is usually referred to as molecular fluctuations. The KS entropy is the number of bits or digits needed to record the time series of the particle coordinates and thus characterizes the information content of the history or path of the system. From this viewpoint, the KS entropy per unit time is closely related to early concepts from Shannon's theory of communication concerned by the transmission and record of information [9].

For continuous stochastic processes such as Brownian motion, dynamical randomness can be characterized by the $(\varepsilon, \tau)$-entropy per unit time, which generalizes the KS entropy per unit time $[10,11]$. The comparison between different random processes has confirmed that the higher the entropy per unit time, the higher the temporal disorder. This is a natural idea since the entropy per unit time is the number of random choices made by the system at each time step. For instance, the game of dice with an entropy per unit time equal to $h=\ln 6$ has more temporal disorder than the coin tossing process with $h=\ln 2$.

Beyond these considerations on the dynamical randomness of the time evolution of a system, each history of the system can be compared with the corresponding time-reversed history. The temporal disorder of the latter can be evaluated in terms of the newly introduced time-reversed entropy per unit time [12]. This quantity gives the information content of the time-reversed histories averaged over the probabilities of the histories themselves. The time-reversed entropy per unit time measures the presence of segments of time-reversed histories among the histories of the forward process. This allows a comparison between the temporal disorders of the process viewed forward or backward in time.

The purpose of the present paper is to show that these concepts are directly related to the thermodynamic entropy production, i.e., to the increase of spatial disorder characterized by the thermodynamic entropy per unit volume. A new interpretation of the second law of thermodynamics is thus provided in terms of temporal disorder instead of spatial disorder. In Section 2, the conceptual framework is set up and the entropies per unit time are defined. The connection to the thermodynamic entropy production is established in Section 3 . The theorem of temporal ordering is stated in Section 4. Section 5 is devoted to the characterization of the large-deviation properties of nonequilibrium fluctuations and the connections with quantities which have been previously introduced in dynamical systems theory. Conclusions are drawn in Section 6.

\section{Temporal disorder of histories and their time reversal}

We consider a process evolving in time. Some observable is recorded at regular time intervals $\Delta t=\tau$. The observable can take several discrete values $\omega_{j} \in\{0,1,2, \ldots, M-1\}$ at each time step $t_{j}=j \tau$ with an integer $j \in \mathbb{Z}$. The sequence of values at successive time steps defines a history or path of the system. If the observation extends from the past to the future, the history or path forms a bi-infinite sequence. If the observation extends over a finite time $t=n \Delta t=n \tau$, the history or path is finite: 


$$
\boldsymbol{\omega}=\omega_{0} \omega_{1} \omega_{2} \cdots \omega_{n-2} \omega_{n-1}=\sigma_{0} \stackrel{\rho_{1}}{\longrightarrow} \sigma_{1} \stackrel{\rho_{2}}{\longrightarrow} \sigma_{2} \stackrel{\rho_{3}}{\longrightarrow} \cdots \stackrel{\rho_{m}}{\longrightarrow} \sigma_{m}
$$

The history or path may consist in a succession of passages across transition states $\rho_{k}$ located between the states $\sigma_{k-1}$ and $\sigma_{k}$ with $k=1,2, \ldots, m$. We notice that the transitions times $t_{k}$ do not necessarily coincide with the sampling times $t_{j}=j \tau$ and, in general, they are not separated by equal time intervals.

If the process is regular, for instance periodic, the number of possible paths is finite and independent of the observation time $t=n \tau$. However, the number of possible paths increases with the observation time $t=n \tau$ if the process presents some randomness. If all the transitions between the different values $\omega_{j} \in\{0,1,2, \ldots, M-1\}$ are possible, the total number of paths of length $n$ is equal to $M^{n}$. For $M=2$, the tree of possible paths is given by

$$
\begin{aligned}
& t=1 \tau: \quad 0,1 \\
& t=2 \tau: \quad 00,01,10,11 \\
& t=3 \tau: \quad 000,001,010,011,100,101,110,111
\end{aligned}
$$

However, even if all these paths are possible, some may occur more often than other and it is important to know their probability:

$$
P(\boldsymbol{\omega})=P\left(\omega_{0} \omega_{1} \omega_{2} \ldots \omega_{n-1}\right)
$$

These considerations apply to deterministic dynamical systems given by a flow $\boldsymbol{\Phi}^{t}$ defined in some phase space $\Gamma$. The different values of the outcome of the observation are the labels of disjoint cells $\mathcal{C}_{\omega}$ forming some partition $\mathcal{P}=\left\{\mathcal{C}_{0}, \mathcal{C}_{1}, \mathcal{C}_{2}, \ldots, \mathcal{C}_{M-1}\right\}$ of the phase space. This partition is also called a coarse graining. A history or path of the system is the sequence of phase-space cells visited at the successive times $t_{j}=j \tau$. The probability (3) of a path is thus given by

$$
P(\boldsymbol{\omega})=P\left(\omega_{0} \omega_{1} \omega_{2} \ldots \omega_{n-1}\right)=P\left(\mathcal{C}_{\omega_{0}} \cap \boldsymbol{\Phi}^{-\tau} \mathcal{C}_{\omega_{1}} \cap \ldots \cap \boldsymbol{\Phi}^{-(n-1) \tau} \mathcal{C}_{\omega_{n-1}}\right)
$$

in terms of the probability density $p_{0}(\Gamma)$ at the initial time: $P(\mathcal{A})=\int_{\mathcal{A}} p_{0}(\Gamma) d \Gamma$ for any set $\mathcal{A}$ in the phase space.

For a quantum dynamical system, the time evolution is ruled by the evolution unitary operator $\hat{U}^{t}=\exp (-i \hat{H} t / \hbar)$ where $\hat{H}$ is the time-independent Hamiltonian. We denote by $\hat{\rho}_{0}$ the density matrix at the initial time and by $\left\{\hat{P}_{\omega}\right\}$ a set of projection operators satisfying $\hat{P}_{\omega} \hat{P}_{\omega^{\prime}}=\hat{P}_{\omega} \delta_{\omega \omega^{\prime}}$ and $\hat{P}_{\omega}^{\dagger}=\hat{P}_{\omega}$. The probability (3) of a path can be defined as

$$
P(\boldsymbol{\omega})=P\left(\omega_{0} \omega_{1} \omega_{2} \ldots \omega_{n-1}\right)=\operatorname{tr}\left(\hat{P}_{\omega_{n-1}} \hat{U}^{\tau} \cdots \hat{P}_{\omega_{1}} \hat{U}^{\tau} \hat{P}_{\omega_{0}} \hat{\rho}_{0} \hat{P}_{\omega_{0}} \hat{U}^{-\tau} \hat{P}_{\omega_{1}} \cdots \hat{U}^{-\tau} \hat{P}_{\omega_{n-1}}\right)
$$

We point out that the classical and quantum dynamical systems may be infinite and composed of unbounded energy or particle reservoirs, in which cases the definition of the path probabilities may require an infinite-system limit.

The dynamical randomness of the process has for consequence that the probability is distributed on a growing number of different paths as time increases. Accordingly, the path probability (3) decreases as the observation time $n \tau$ increases. In the case of random processes, the decrease is typically exponential which motivates the introduction of the entropy per unit time:

$$
h \equiv \lim _{n \rightarrow \infty}-\frac{1}{n \tau} \sum_{\boldsymbol{\omega}} P(\boldsymbol{\omega}) \ln P(\boldsymbol{\omega})=\lim _{n \rightarrow \infty}-\frac{1}{n \tau} \sum_{\omega_{0} \omega_{1} \omega_{2} \ldots \omega_{n-1}} P\left(\omega_{0} \omega_{1} \omega_{2} \ldots \omega_{n-1}\right) \ln P\left(\omega_{0} \omega_{1} \omega_{2} \ldots \omega_{n-1}\right)
$$

For the coin tossing process, the path probabilities decrease as $2^{-n}$ so that this quantity takes the value $h=\ln 2$ if there is a toss every time unit. For the game of dice, the decrease of the path probabilities goes as $6^{-n}$ and the dynamical entropy is $h=\ln 6$. The entropy per unit time gives the rate of growth of the tree of possible typical paths of the random process. By typical paths, we mean the paths occurring $N(\boldsymbol{\omega})=N_{\text {tot }} P(\boldsymbol{\omega})$ times in a sample of $N_{\text {tot }}$ sequences of length $n$. The typical paths form a subset of $\exp (h n \tau)$ paths among the complete set of $M^{n}$ possible sequences so that the entropy per unit time is bounded according to $h \leq(\ln M) / \tau$. The equality is satisfied in the case where all the possible paths are equally probable. Since the number of typical paths is lower than the number of possible paths, the observation of the process over the time $t=n \tau$ should require a memory of $h n \tau \log _{2}$ e bits, instead of $n \log _{2} M$ bits. Indeed, the record of the observed path is carried out by giving an integer number in the list of the $\exp (h n \tau)$ typical paths, rather than in the list of all the $M^{n}$ possible paths. Therefore, a compression of information can be obtained by taking into account the probabilities of occurrences of the different 
paths. The entropy per unit time thus gives the minimum number of nats (or bits if we use logarithms to the base 2 ) needed to faithfully record the paths of the process. The entropy per unit time can be interpreted as the rate of production of information by the random process: the larger the entropy per unit time, the higher the dynamical randomness. In this sense, it measures the temporal disorder of the process along the time axis.

This interpretation is supported by the comparison between different random processes [10]. In classical dynamical systems, the KS entropy per unit time is defined as the supremum of $h$ over all the possible partitions $\mathcal{P}$. According to Pesin's theorem [5,13], the KS entropy is equal to the sum of positive Lyapunov exponents in hyperbolic systems, which expresses the fact that dynamical randomness directly finds its origin in the sensitivity to initial conditions. The existence of a supremum means that the entropy per unit time is finite in classical dynamical systems where the random choices are discrete at each time step. However, the KS entropy is infinite in random processes with continuous random variables. In this case, the entropy per unit time (6) is still well defined and the random process is characterized by the way its entropy per unit time increases to infinity as the sampling time $\tau$ or the size $\varepsilon$ of the cells of the partition go to zero [10]. The entropy per unit time can be arbitrarily large for small enough values of $\tau$ or $\varepsilon$, which means that the temporal disorder of these random processes is higher than in classical dynamical systems. This expresses quantitatively our intuition that the random signal of Brownian motion is much more irregular than a trajectory of the Lorenz or Rössler chaotic attractors, for instance.

An analogy exists with the thermodynamic entropy per unit length in a polymer or the thermodynamic entropy per unit volume in bulk matter at equilibrium. Indeed, these usual thermodynamic entropies are also defined by Eq. (6) where $n \tau$ denotes the length or the volume and $P$ is the equilibrium probability distribution. In these cases, the entropy per unit length or unit volume is a measure of the spatial disorder in the system at equilibrium as it should be, according to Boltzmann's interpretation of the thermodynamic entropy. With space replaced by time, the entropy per unit time (6) thus characterizes the temporal disorder of time-evolution processes in much the same way as the usual thermodynamic entropy does for spatial disorder.

Now, we may wonder what is the probability of occurrence of paths formed by time reversing the paths of the process. The time-reversed path corresponding to the path (1) is given by

$$
\boldsymbol{\omega}^{\mathrm{R}}=\omega_{n-1} \omega_{n-2} \cdots \omega_{2} \omega_{1} \omega_{0}=\sigma_{m} \stackrel{\rho_{m}}{\longrightarrow} \cdots \stackrel{\rho_{3}}{\longrightarrow} \sigma_{2} \stackrel{\rho_{2}}{\longrightarrow} \sigma_{1} \stackrel{\rho_{1}}{\longrightarrow} \sigma_{0}
$$

We emphasize that the time-reversed paths are in general different from the corresponding paths even if the underlying deterministic dynamical system is time-reversal symmetric. Moreover, the probability weight given to a time-reversed path may differ from the probability weight of the corresponding path. Indeed, the Newtonian scheme has historically acquired the time-reversal symmetry at the price of leaving free the initial conditions. It remains usually unnoticed that the selection of initial conditions breaks in general the time-reversal symmetry. Indeed, this selection amounts to weighting the selected trajectory by a probability equal to one while all the other trajectories have zero probability. The selection of initial conditions can break the time-reversal symmetry even in a simple system such as a particle in uniform motion with respect to an inertial frame. In this case, Newton's equation is $d^{2} x / d t^{2}=0$ and the selected trajectory is the line at velocity $v=v_{0}$ in the phase space $(x, v)$. This phase-space line differs from the time-reversed trajectory at $v=-v_{0}$ if $v_{0} \neq 0$. Weighting the former and not the latter with a unit probability thus breaks the time-reversal symmetry in the same way as other symmetries are broken in condensed matter physics.

As a consequence, we should expect that, in general, the probability of the time-reversed path (7) is different from the probability of the path (1). They may thus decrease at different rates as the time $t=n \tau$ increases, meaning that they have different information contents. In order to characterize this difference, we have recently introduced the time-reversed entropy per unit time [12]

$$
h^{\mathrm{R}} \equiv \lim _{n \rightarrow \infty}-\frac{1}{n \tau} \sum_{\boldsymbol{\omega}} P(\boldsymbol{\omega}) \ln P\left(\boldsymbol{\omega}^{\mathrm{R}}\right)=\lim _{n \rightarrow \infty}-\frac{1}{n \tau} \sum_{\omega_{0} \omega_{1} \omega_{2} \ldots \omega_{n-1}} P\left(\omega_{0} \omega_{1} \omega_{2} \ldots \omega_{n-1}\right) \ln P\left(\omega_{n-1} \ldots \omega_{2} \omega_{1} \omega_{0}\right)
$$

beside the entropy per unit time (6). This quantity is defined by averaging with respect to the probabilities of the paths themselves. If the average was taken with the probabilities of the time-reversed paths, the quantity would be identical to the entropy per unit time (6). We point out that the time-reversed entropy per unit time should be defined in terms of the probabilities of the time-reversed paths for the time-reversed process if this latter is distinct from the process [14].

We should have that

$$
\begin{gathered}
P(\boldsymbol{\omega})=P\left(\omega_{0} \omega_{1} \omega_{2} \ldots \omega_{n-1}\right) \sim \exp (-h n \tau) \\
P\left(\boldsymbol{\omega}^{\mathrm{R}}\right)=P\left(\omega_{n-1} \ldots \omega_{2} \omega_{1} \omega_{0}\right) \sim \exp \left(-h^{\mathrm{R}} n \tau\right)
\end{gathered}
$$


for almost all paths $\boldsymbol{\omega}$ with respect to the probability measure $P$ of an ergodic process. This is the ShannonMcMillan-Breiman theorem [15] completed with a similar property for the corresponding time-reversed paths. If the entropy per unit time $h$ measures the temporal disorder of the process, the time-reversed entropy per unit time $h^{\mathrm{R}}$ measures the temporal disorder among the time-reversed paths of the process. In this way, the difference between the quantities $h$ and $h^{\mathrm{R}}$ characterizes the breaking of the time-reversal symmetry in the temporal disorder of the process.

\section{Temporal disorder and thermodynamic entropy production}

The previous concepts turn out to be related to the thermodynamic entropy production in a nonequilibrium steady state. We here consider processes in which the sequences of states $\omega_{j}$ are Markovian over the sampling time $\tau$. In this regard, we notice that, in some classical dynamical systems, it is possible to construct partitions of the phase space in such a way that the process is indeed Markovian [6,16]. There also exist many examples of classical and quantum dynamical systems which are described with very good approximation by Markovian processes ruled by the Pauli master equation:

$$
\frac{d}{d t} P_{t}(\omega)=\sum_{\omega^{\prime}(\neq \omega)}\left[P_{t}\left(\omega^{\prime}\right) W\left(\omega^{\prime} \mid \omega\right)-P_{t}(\omega) W\left(\omega \mid \omega^{\prime}\right)\right]
$$

for the probability $P_{t}(\omega)$ to find the system in the state $\omega$ by the time $t . W\left(\omega \mid \omega^{\prime}\right)$ denotes the rate of the transition $\omega \rightarrow \omega^{\prime}$. These states can be defined as the succession of a transition state $\rho$ and a state $\sigma: \omega=\rho \sigma$. We recover the general form of the master equation [2] if we define the probability to find the system in the state $\sigma$ as $P_{t}(\sigma)=\sum_{\rho} P_{t}(\rho \sigma)$ and assume that the transition rates are independent of the previous transition state $W\left(\rho^{\prime} \sigma^{\prime} \mid \rho \sigma\right)=W_{\rho}\left(\sigma^{\prime} \mid \sigma\right)$.

In this framework, the thermodynamic entropy can be defined as

$$
S=\sum_{\omega} P_{t}(\omega) S^{0}(\omega)-k_{\mathrm{B}} \sum_{\omega} P_{t}(\omega) \ln P_{t}(\omega)
$$

which characterizes disorder in the probability distribution $P_{t}(\omega)[18,19] . S^{0}(\omega)$ denotes the entropy associated with the disorder over the microscopic degrees of freedom if the system is in the coarse-grained state $\omega . k_{\mathrm{B}}$ is Boltzmann's constant. Since the probability distribution $P_{t}(\omega)$ evolves in time according to the master equation (11), the time derivative of the entropy can be written as follows $[18,19]$

$$
\frac{d S}{d t}=\frac{d_{\mathrm{e}} S}{d t}+\frac{d_{\mathrm{i}} S}{d t}
$$

in terms of the thermodynamic entropy flow

$$
\frac{d_{\mathrm{e}} S}{d t}=-\sum_{\omega \neq \omega^{\prime}} J_{t}\left(\omega \mid \omega^{\prime}\right)\left[S^{0}(\omega)+\frac{k_{\mathrm{B}}}{2} \ln \frac{W\left(\omega \mid \omega^{\prime}\right)}{W\left(\omega^{\prime} \mid \omega\right)}\right]
$$

and the thermodynamic entropy production

$$
\frac{d_{\mathrm{i}} S}{d t}=\frac{1}{2} \sum_{\omega \neq \omega^{\prime}} J_{t}\left(\omega \mid \omega^{\prime}\right) A_{t}\left(\omega \mid \omega^{\prime}\right) \geq 0
$$

In Eqs. (14) and (15), we have introduced the probability current between the states $\omega$ and $\omega^{\prime}$ as

$$
J_{t}\left(\omega \mid \omega^{\prime}\right) \equiv P_{t}(\omega) W\left(\omega \mid \omega^{\prime}\right)-P_{t}\left(\omega^{\prime}\right) W\left(\omega^{\prime} \mid \omega\right)
$$

and the corresponding affinity as

$$
A_{t}\left(\omega \mid \omega^{\prime}\right) \equiv k_{\mathrm{B}} \ln \frac{P_{t}(\omega) W\left(\omega \mid \omega^{\prime}\right)}{P_{t}\left(\omega^{\prime}\right) W\left(\omega^{\prime} \mid \omega\right)}
$$

In a steady state, the probabilities of the states $\omega$ are constant, $d P(\omega) / d t=0$, and the probability of a path can be calculated by

$$
P(\boldsymbol{\omega})=P\left(\omega_{0} \omega_{1} \omega_{2} \ldots \omega_{n-1}\right)=P\left(\omega_{0}\right) P\left(\omega_{0} \mid \omega_{1}\right) P\left(\omega_{1} \mid \omega_{2}\right) \cdots P\left(\omega_{n-2} \mid \omega_{n-1}\right)
$$


where the conditional probabilities of finding the system in the state $\omega_{j+1}$ provided that it was in the state $\omega_{j}$ at the preceding time step are given by

$$
P\left(\omega_{j} \mid \omega_{j+1}\right)= \begin{cases}W\left(\omega_{j} \mid \omega_{j+1}\right) \tau+O\left(\tau^{2}\right) & \text { if } \omega_{j} \neq \omega_{j+1} \\ 1-\sum_{\omega^{\prime}\left(\neq \omega_{j}\right)} W\left(\omega_{j} \mid \omega^{\prime}\right) \tau+O\left(\tau^{2}\right) & \text { if } \omega_{j}=\omega_{j+1}\end{cases}
$$

for $\tau \rightarrow 0$.

For this continuous-time jump process, the entropy per unit time (6) is given by [10]

$$
h=\left(\ln \frac{\mathrm{e}}{\tau}\right) \sum_{\omega \neq \omega^{\prime}} P(\omega) W\left(\omega \mid \omega^{\prime}\right)-\sum_{\omega \neq \omega^{\prime}} P(\omega) W\left(\omega \mid \omega^{\prime}\right) \ln W\left(\omega \mid \omega^{\prime}\right)+O(\tau)
$$

and the time-reversed entropy per unit time $(8)$ by $[12,17]$

$$
h^{\mathrm{R}}=\left(\ln \frac{\mathrm{e}}{\tau}\right) \sum_{\omega \neq \omega^{\prime}} P(\omega) W\left(\omega \mid \omega^{\prime}\right)-\sum_{\omega \neq \omega^{\prime}} P(\omega) W\left(\omega \mid \omega^{\prime}\right) \ln W\left(\omega^{\prime} \mid \omega\right)+O(\tau)
$$

We notice that the term in (lne) in each one of both expressions comes from the contribution of the conditional probabilities (19) with $\omega_{j}=\omega_{j+1}$. The entropies per unit time (20) and (21) differ by the exchange of $\omega$ and $\omega^{\prime}$ in the transition rate appearing in the logarithm. In a steady state, their difference turns out to be equal to the thermodynamic entropy production (15)

$$
\lim _{\tau \rightarrow 0}\left(h^{\mathrm{R}}-h\right)=\frac{1}{2} \sum_{\omega \neq \omega^{\prime}}\left[P(\omega) W\left(\omega \mid \omega^{\prime}\right)-P\left(\omega^{\prime}\right) W\left(\omega^{\prime} \mid \omega\right)\right] \ln \frac{P(\omega) W\left(\omega \mid \omega^{\prime}\right)}{P\left(\omega^{\prime}\right) W\left(\omega^{\prime} \mid \omega\right)}=\frac{1}{k_{\mathrm{B}}} \frac{d_{\mathrm{i}} S}{d t}
$$

in the limit where the sampling time $\tau$ is small enough. This relationship holds for continuous-time jump processes [12], as well as for classical deterministic systems in the escape-rate and thermostated-system approaches [20]. Closely related results have recently been obtained $[21,22,23,24]$.

\section{Temporal ordering out of equilibrium}

According to Eq. (22), the thermodynamic entropy production can be written with the definitions (6) and (8) as the relative entropy of the path probabilities with respect to the probabilities of the time-reversed paths:

$$
\begin{aligned}
\frac{1}{k_{\mathrm{B}}} \frac{d_{\mathrm{i}} S}{d t} & =\lim _{\tau \rightarrow 0}\left(h^{\mathrm{R}}-h\right) \\
& =\lim _{\tau \rightarrow 0} \lim _{n \rightarrow \infty} \frac{1}{n \tau} \sum_{\boldsymbol{\omega}} P(\boldsymbol{\omega}) \ln \frac{P(\boldsymbol{\omega})}{P\left(\boldsymbol{\omega}^{\mathrm{R}}\right)} \\
& =\lim _{\tau \rightarrow 0} \lim _{n \rightarrow \infty} \frac{1}{2 n \tau} \sum_{\boldsymbol{\omega}}\left[P(\boldsymbol{\omega})-P\left(\boldsymbol{\omega}^{\mathrm{R}}\right)\right] \ln \frac{P(\boldsymbol{\omega})}{P\left(\boldsymbol{\omega}^{\mathrm{R}}\right)} \geq 0
\end{aligned}
$$

which is always non-negative [12]. The reason is that $(x-y) \ln (x / y) \geq 0$ for any non-negative real numbers $x$ and $y$ so that we have the general inequality

$$
h^{\mathrm{R}} \geq h
$$

Since $(x-y) \ln (x / y)=0$ if and only if $x=y$, we infer that the equality $h^{\mathrm{R}}=h$ holds in the equilibrium state where the entropy production vanishes. Indeed, the condition of detailed balance is satisfied at equilibrium so that the probability of each path tends to be equal to the probability of the corresponding time-reversed path in the long-time limit $n \rightarrow \infty$. In this case, the entropy per unit time becomes equal to the time-reversed entropy per unit time.

In contrast, in a nonequilibrium steady state, we infer that the time-reversed entropy per unit time is larger than the entropy per unit time: $h^{\mathrm{R}}>h$. With the interpretation of these quantities in terms of temporal disorder given in Section 2, we see that, in a nonequilibrium steady state, the temporal disorder of the paths is smaller than the temporal disorder of the corresponding time-reversed paths in the sense that the probabilities of the time-reversed paths decrease faster than for the paths themselves. Therefore, the time-reversed paths are rarer than the paths 


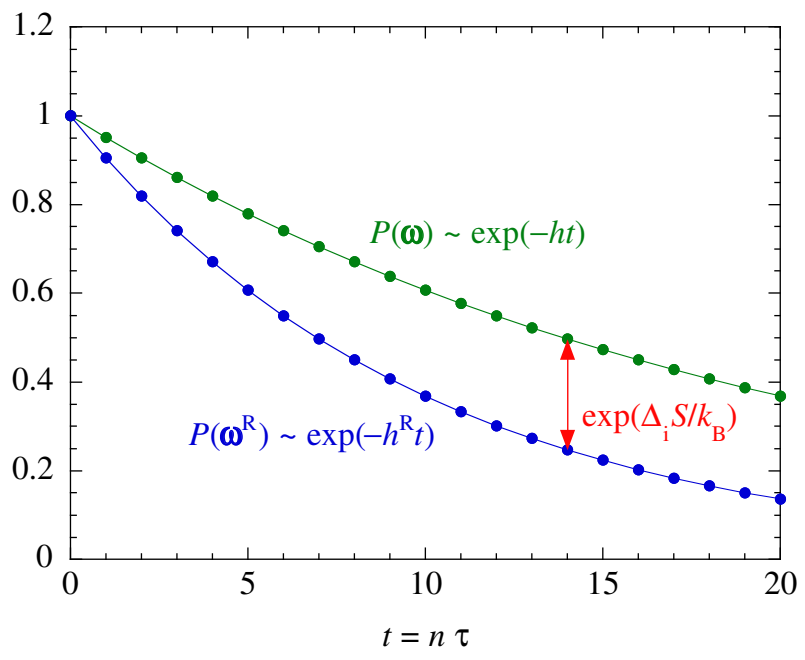

Figure 1. Diagram showing the probability of a path and the probability of the corresponding time reversal as a function of the number $n$ of time intervals $\tau$, illustrating the formula (22). $\Delta_{\mathrm{i}} S$ denotes the entropy production during the whole duration $n \tau$.

in a nonequilibrium steady state as illustrated in Fig. 1. This results into a phenomenon of temporal ordering as the system is driven out of equilibrium and we have the

Theorem of nonequilibrium temporal ordering: In nonequilibrium steady states, the typical paths are more ordered in time than their corresponding time reversals in the sense that their temporal disorder characterized by $h$ is smaller than the temporal disorder of the corresponding time-reversed paths characterized by $h^{\mathrm{R}}$.

This theorem expresses the fact that the molecular fluctuations such as Brownian motion are completely erratic at equilibrium, albeit they acquire a privileged direction and are thus more ordered out of equilibrium. The formula (22) shows that the thermodynamic entropy production finds its origin in a time asymmetry between the probabilities of each path and its corresponding time reversal. The temporal ordering is compatible with Boltzmann's interpretation of the second law according to which spatial disorder increases with time. The temporal ordering is possible at the expense of an increase in the spatial disorder.

The theorem also gives a dynamical information-theoretic interpretation to the second law of thermodynamics and opens far reaching perspectives. The nonequilibrium temporal ordering can be illustrated with the process of erasure of a computer memory [25,26]. Let us suppose that the memory of a computer contains a long arbitrary sequence of bits 0 or 1 and an erasing device successively deletes them at a rate of one bit per unit time. This erasing process does not generate new information and its entropy per unit time is thus vanishing: $h=0$. In contrast, the time-reversed process produces information at a rate of one new bit per unit time so that the timereversed entropy per unit time is equal to $h^{\mathrm{R}}=\ln 2$. According to Eq. (22), the process of erasing one bit per unit time should thus have a thermodynamic entropy production equal to $d_{\mathrm{i}} S / d t=k_{\mathrm{B}}\left(h^{\mathrm{R}}-h\right)=k_{\mathrm{B}} \ln 2$, in agreement with Landauer's and Bennett's conclusion $[25,26]$. This example shows that the process has a greater temporal order than its time reversal.

\section{Large-deviation properties of nonequilibrium fluctuations}

In this section, our purpose is to investigate the large-deviation properties of the probabilities $P(\boldsymbol{\omega})$ and $P\left(\boldsymbol{\omega}^{\mathrm{R}}\right)$ in the long-time limit $n \rightarrow \infty$. In this way, connections will be established with large-deviation functions introduced in the eighties in the study of chaotic dynamical systems and, more recently, in the study of the so-called fluctuation relations.

The average with respect to the path probabilities is denoted by $\langle\cdot\rangle=\sum_{\boldsymbol{\omega}} P(\boldsymbol{\omega})(\cdot)$. We introduce the generating function of both the forward and backward local entropies per unit time as 


$$
J(\alpha, \beta) \equiv \lim _{t \rightarrow \infty}-\frac{1}{t} \ln \left\langle P(\boldsymbol{\omega})^{\alpha} P\left(\boldsymbol{\omega}^{\mathrm{R}}\right)^{\beta}\right\rangle=\lim _{t \rightarrow \infty}-\frac{1}{t} \ln \sum_{\boldsymbol{\omega}} P(\boldsymbol{\omega})^{\alpha+1} P\left(\boldsymbol{\omega}^{\mathrm{R}}\right)^{\beta}
$$

with $t=n \tau$. The standard and time-reversed entropies per unit time are given in the Taylor expansion of $J(\alpha, \beta)$ around $\alpha=\beta=0$ :

$$
\begin{aligned}
J(0,0) & =0 \\
\frac{\partial J}{\partial \alpha}(0,0) & =h \\
\frac{\partial J}{\partial \beta}(0,0) & =h^{\mathrm{R}}
\end{aligned}
$$

Moreover, this generating function satisfies the symmetry

$$
J(\alpha, \beta)=J(\beta-1, \alpha+1)
$$

as the consequence of the fact that the sum over the paths can be replaced by the sum over the time-reversed paths in the definition of the generating function.

The ratio of the forward to backward probabilities gives the quantity introduced by Lebowitz and Spohn [27] as

$$
Z(t) \simeq \ln \frac{P(\boldsymbol{\omega})}{P\left(\boldsymbol{\omega}^{\mathrm{R}}\right)}
$$

in the long-time limit $t \rightarrow \infty$. The generating function of this quantity is given by

$$
Q(\eta) \equiv \lim _{t \rightarrow \infty}-\frac{1}{t} \ln \left\langle\mathrm{e}^{-\eta Z(t)}\right\rangle=J(-\eta, \eta)
$$

which thus satisfies

$$
\begin{aligned}
Q(0) & =J(0,0)=0 \\
\frac{d Q}{d \eta}(0) & =\lim _{t \rightarrow \infty} \frac{\langle Z(t)\rangle}{t}=-\frac{\partial J}{\partial \alpha}(0,0)+\frac{\partial J}{\partial \beta}(0,0)=h^{\mathrm{R}}-h
\end{aligned}
$$

In a nonequilibrium steady state, it is known that the linear growth with time of the quantity $Z(t)$ gives the mean entropy production according to

$$
\lim _{t \rightarrow \infty} \frac{\langle Z(t)\rangle}{t}=\frac{1}{k_{\mathrm{B}}} \frac{d_{\mathrm{i}} S}{d t}
$$

in consistency with the formula (22). Furthermore, the symmetry relation (31) gives the relation

$$
Q(\eta)=Q(1-\eta)
$$

of the fluctuation theorem $[27,28,29,30,31,32,33,34,35,36,37,38,39,40,41]$. Consequently, the symmetry relation (31) extends the fluctuation theorem to the generating function (27) of the forward and backward local entropies per unit time.

On the other hand, Grassberger and Procaccia [42] introduced the Renyi entropy per unit time as

$$
K(q) \equiv \frac{1}{1-q} \lim _{t \rightarrow \infty} \frac{1}{t} \ln \sum_{\boldsymbol{\omega}} P(\boldsymbol{\omega})^{q}
$$

The comparison with Eq. (27) shows that

$$
K(q)=\frac{1}{q-1} J(q-1,0)=\frac{1}{q-1} J(-1, q)
$$

so that the generating function (27) contains the Renyi entropy per unit time. We recover the result that the Renyi entropy per unit time at $q=1$ is equal to the entropy per unit time itself $K(1)=h$. At $q=0$, it gives the growth rate of the total number of possible paths, $K(0)=(\ln M) / \tau$ (which corresponds to the topological entropy per unit time in chaotic dynamical systems). The Renyi entropy is related by a Legendre transform to a function introduced by Eckmann and Procaccia [43] according to

$$
G(\lambda)=q \lambda-(q-1) K(q)=q \lambda-J(q-1,0)
$$

with

$$
q=\frac{d G}{d \lambda}(\lambda) \quad \text { and } \quad \lambda=\frac{\partial J}{\partial \alpha}(q-1,0)
$$


At $q=1$, we have that

$$
h=\lambda(1)=G[\lambda(1)]=G(h) \quad \text { and } \quad \frac{d G}{d \lambda}(h)=1
$$

The second derivative of $G(\lambda)$ - if it exists - gives the diffusivity coefficient

$$
D=\lim _{t \rightarrow \infty} \frac{1}{2 t}\left\langle[\ln P(\boldsymbol{\omega})+h t]^{2}\right\rangle=-\frac{1}{2 \frac{d^{2} G}{d \lambda^{2}}(h)}=-\frac{1}{2} \frac{\partial^{2} J}{\partial \alpha^{2}}(0,0)
$$

entering in a central limit theorem for mixing processes obtained by Ibragimov [44].

Now, a large-deviation function can be defined as the growth rate of the probability that the forward and backward local entropies per unit time take given values $\lambda$ and $\mu$ :

$$
L(\lambda, \mu) \equiv \lim _{t \rightarrow \infty} \frac{1}{t} \ln P\left\{\boldsymbol{\omega}:-\frac{1}{t} \ln P(\boldsymbol{\omega}) \in(\lambda, \lambda+d \lambda) \&-\frac{1}{t} \ln P\left(\boldsymbol{\omega}^{\mathrm{R}}\right) \in(\mu, \mu+d \mu)\right\}
$$

This function is related to the generating function (27) by the Legendre transform:

$$
\begin{aligned}
& J(\alpha, \beta)=\operatorname{Min}_{\lambda, \mu}[\alpha \lambda+\beta \mu-L(\lambda, \mu)] \\
& L(\lambda, \mu)=\operatorname{Min}_{\alpha, \beta}[\alpha \lambda+\beta \mu-J(\alpha, \beta)]
\end{aligned}
$$

and it reaches its maximum at the point $\lambda=h$ and $\mu=h^{\mathrm{R}}$ :

$$
\begin{aligned}
L\left(h, h^{\mathrm{R}}\right) & =0 \\
\frac{\partial L}{\partial \lambda}\left(h, h^{\mathrm{R}}\right) & =0 \\
\frac{\partial L}{\partial \mu}\left(h, h^{\mathrm{R}}\right) & =0
\end{aligned}
$$

with $h^{\mathrm{R}}=h+\left(1 / k_{\mathrm{B}}\right)\left(d_{\mathrm{i}} S / d t\right)$. If the second derivatives exist, the forward and backward local entropies per unit time, $-(1 / t) \ln P(\boldsymbol{\omega})$ and $-(1 / t) \ln P\left(\boldsymbol{\omega}^{\mathrm{R}}\right)$, obey a central limit theorem showing that these quantities have Gaussian fluctuations around their mean values $h$ and $h^{\mathrm{R}}$ under these conditions.

\section{Conclusions}

In this paper, we have shown how the properties of dynamical randomness of the paths and their corresponding time reversal are connected to the thermodynamic entropy production.

Dynamical randomness is characterized by the decrease of the path probabilities. The rate of decrease is an entropy per unit time which measures the temporal disorder of the process. Similar considerations apply to the time-reversed paths. In nonequilibrium steady states, the probabilities of the time-reversed paths differ in general from the path probabilities and they decrease at different rates. These considerations motivate the introduction of a concept of time-reversed entropy per unit time, which characterizes temporal disorder in the time-reversed paths. The central result is that the difference between the time-reversed and standard entropies per unit time gives the thermodynamic entropy production. A related result has been experimentally verified for a driven Brownian particle and a nonvanishing fluctuating electric current across a resistor [14].

In this perspective, a new dynamical information-theoretic interpretation can be given to the second law of thermodynamics. At equilibrium, the temporal disorders of the paths and their time reversal are equal and the entropy production vanishes. Out of equilibrium, the temporal disorder of the time-reversed paths turns out to be larger than the temporal disorder of the paths themselves in direct relationship with the positive thermodynamic entropy production. In this way, a theorem can be established according to which the temporal order of the paths is greater than for the time-reversed paths, as a corollary of the second law of thermodynamics. This reflects the time asymmetry in the stationary probability measure of the nonequilibrium steady state. The nonequilibrium constraints on the system break in general the time-reversal symmetry at the level of the statistical description in terms of a probability measure even if the underlying microscopic dynamics has the symmetry. The present result shows that the thermodynamic entropy production can be considered as the parameter of temporal order in the out-of-equilibrium breaking of the time-reversal symmetry. Most remarkably, Boltzmann's increase of spatial disorder turns out to generate temporal ordering. 
The relationships between the thermodynamic entropy production and the temporal disorder of the nonequilibrium fluctuations observed forward or backward in time can be further characterized in terms of their largedeviation properties. In this way, we have obtained fundamental relationships between large-deviation functions introduced in different contexts.

\section{Acknowledgements}

This research is financially supported by the F. N. R. S. Belgium and the "Communauté française de Belgique" (contract "Actions de Recherche Concertées" No. 04/09-312).

\section{References}

[1] D. A. McQuarrie and J. D. Simon, Physical Chemistry: A Molecular Approach (University Science Books, Sausalito, 1997).

[2] G. Nicolis and I. Prigogine, Self-Organization in Nonequilibrium Systems (Wiley, New York, 1977).

[3] A. N. Kolmogorov, Dokl. Akad. Nauk SSSR 124, 754 (1959).

[4] Ya. G. Sinai, Dokl. Akad. Nauk SSSR 124, 768 (1959).

[5] J.-P. Eckmann and D. Ruelle, Rev. Mod. Phys. 57, 617 (1985).

[6] P. Gaspard, Chaos, Scattering, and Statistical Mechanics (Cambridge University Press, Cambridge UK, 1998).

[7] H. van Beijeren, J. R. Dorfman, H. A. Posch, and Ch. Dellago, Phys. Rev. E 56, 5272 (1997).

[8] P. Gaspard and H. van Beijeren, J. Stat. Phys. 109, 671 (2002).

[9] C. Shannon, Bell System Tech. J. 27, 379, 623 (1948).

[10] P. Gaspard and X. J. Wang, Phys. Rep. 235, 321 (1993).

[11] P. Gaspard, M. E. Briggs, M. K. Francis, J. V. Sengers, R. W. Gammon, J. R. Dorfman, and R. V. Calabrese, Nature 394, 865 (1998).

[12] P. Gaspard, J. Stat. Phys. 117, 599 (2004).

[13] Ya. B. Pesin, Russian Math. Surveys 32, 55 (1977).

[14] D. Andrieux, P. Gaspard, S. Ciliberto, N. Garnier, S. Joubaud, and A. Petrosyan, Phys. Rev. Lett. 98, 150601 (2007).

[15] I. P. Cornfeld, S. V. Fomin, and Ya. G. Sinai, Ergodic Theory (Springer, Berlin, 1982).

[16] G. Nicolis and C. Nicolis, Phys. Rev. A 38, 427 (1988).

[17] P. Gaspard, New Journal of Physics 7, 77 (2005).

[18] J. Schnakenberg, Rev. Mod. Phys. 48, 571 (1976).

[19] Luo Jiu-li, C. Van den Broeck, and G. Nicolis, Z. Phys. B - Condensed Matter 56, 165 (1984).

[20] P. Gaspard, to appear in Adv. Chem. Phys. (2007).

[21] V. Lecomte, C. Appert-Rolland, and F. van Wijland, Phys. Rev. Lett. 95, 010601 (2005).

[22] C. Jarzynski, Phys. Rev. E 73, 046105 (2006).

[23] J. Naudts and E. Van der Straeten, Phys. Rev. E 74, 040103R (2006).

[24] R. Kawai, J. M. R. Parrondo, and C. Van den Broeck, Phys. Rev. Lett. 98, 080602 (2007).

[25] R. Landauer, IBM J. Res. Dev. 5, 183 (1961).

[26] C. H. Bennett, Int. J. Theor. Phys. 21, 905 (1982).

[27] J. L. Lebowitz and H. Spohn, J. Stat. Phys. 95, 333 (1999).

[28] D. J. Evans, E. G. D. Cohen, and G. P. Morriss, Phys. Rev. Lett. 71, 2401 (1993).

[29] D. J. Evans and D. J. Searles, Phys. Rev. E 50, 1645 (1994).

[30] G. Gallavotti and E. G. D. Cohen, Phys. Rev. Lett. 74, 2694 (1995).

[31] J. Kurchan, J. Phys. A: Math. Gen. 31, 3719 (1998).

[32] G. E. Crooks, Phys. Rev. E 60, 2721 (1999).

[33] C. Maes, J. Stat. Phys. 95, 367 (1999).

[34] G. M. Wang, E. M. Sevick, E. Mittag, D. J. Searles, and D. J. Evans, Phys. Rev. Lett. 89, 050601 (2002). 
[35] R. van Zon and E. G. D. Cohen, Phys. Rev. Lett. 91, 110601 (2003).

[36] R. van Zon, S. Ciliberto and E. G. D. Cohen, Phys. Rev. Lett. 92, 130601 (2004).

[37] N. Garnier and S. Ciliberto, Phys. Rev. E 71, R060101 (2005).

[38] P. Gaspard, J. Chem. Phys. 120, 8898 (2004).

[39] D. Andrieux and P. Gaspard, J. Chem. Phys. 121, 6167 (2004).

[40] U. Seifert, Europhys. Lett. 70, 36 (2005).

[41] B. Cleuren, C. Van den Broeck, and R. Kawai, Phys. Rev. Lett. 96, 050601 (2006).

[42] P. Grassberger and I. Procaccia, Phys. Rev. A 28, 2591 (1983).

[43] J.-P. Eckmann and I. Procaccia, Phys. Rev. A 34, 659 (1986).

[44] I. A. Ibragimov, Theor. Probability Appl. 7, 349 (1962). 